\title{
Diffusion-Weighted Magnetic Resonance Imaging (DWI) Parameters in Benign and Malignant Ovarian Tumors with Solid and Cystic Components
}

\author{
Yan Zhuang, Tianping Wang and Guofu Zhang
}

\begin{abstract}
Objective: To determine the values of diffusion-weighted magnetic resonance imaging (DWI) parameters on differential diagnosis of benign and malignant ovarian tumours with solid and cystic components.

Study Design: An observational study.

Place and Duration of Study: Department of Radiology, Obstetrics and Gynecology Hospital of Fudan University, China, from January to December 2017.

Methodology: Forty-four cases of malignant and benign ovarian tumours were selected as respective groups. Inclusion criteria were ovarian masses found by pelvic and abdominal imaging examination. Exclusion criteria of two groups were endometriosis and pelvic inflammatory diseases found by relevant examination, those with chronic diseases, tumors in other organs found by relevant examination, and those with family cancer history. Conventional MRI plain scans, enhanced scans and DWI scans, were performed after admission. Apparent diffusion coefficient (ADC) and exponential apparent diffusion imaging (eADC) of solid lesions of two groups were recorded and compared.

Results: Solid ADC in malignant group was lower than that in benign group $(p<0.001)$. Solid eADC in malignant group was higher than that in benign group $(p<0.001)$.

Conclusion: Diffusion-weighted magnetic resonance imaging is of high application value in the differential diagnosis of benign and malignant ovarian tumours with solid and cystic components, and it is worthy of promotion and application.
\end{abstract}

Key Words: Diffusion-weighted magnetic resonance imaging (DWI), Ovarian cyst, Tumour, Benign and malignant, Apparent diffusion coefficient, Diagnosis.

\section{INTRODUCTION}

Ovarian cancer is one of the most common female genital cancers. The incidence is second only to cervical cancer and endometrial cancer, with the highest mortality. 1,2 With the development in medical imaging, magnetic resonance imaging (MRI) has been used gradually in the diagnosis of ovarian diseases. Nishio et al. found that the MRI findings, which suggested malignant transformation, were emergence of a solid portion and increase in cyst size. ${ }^{3}$ But sensitivity of MRI is relatively low in the identification of benign and malignant tumours. ${ }^{4}$

Diffusion-weighted magnetic resonance imaging (DWI) non-invasively reflects the characteristics of molecular diffusion.5,6 DWI is mainly used for the diagnosis of neurological diseases. With the development of magnetic resonance hardware and software, the application of DWI has been extended to abdominal

Dapartment of Radiology, Obstetrics and Gynecology Hospital of Fudan University, Shanghai, 200011, China

Correspondence: Tianping Wang, Dapartment of Radiology, Obstetrics and Gynecology Hospital of Fudan University,

Shanghai, 200011, China

E-mail: zhuangy0808@sina.com

Received: April 16, 2018; Accepted: September 29, 2018 diseases. ${ }^{7}$ Razik et al. pointed out that assessment of the morphology of the reactive tumor stalk on DWI has better diagnostic performance in predicting muscle invasion in urinary bladder cancer than conventional MRI. ${ }^{8}$ Because DWI is very sensitive to water molecular movement, it is not subject to respiratory movement, heartbeat and pulse, peristalsis and other effects, and has been successfully used in abdominal and pelvic imaging examinations, enhancing the role and potential of MRI in the evaluation of female pelvic lesions. ${ }^{9}$ Duarte et al. revealed the most relevant physiological and benign pathological conditions of the female pelvis that could show restricted diffusion on DWI. 10

The objective of this study was to determine the values of diffusion-weighted magnetic resonance imaging (DWI) parameters on the differential diagnosis of benign and malignant ovarian tumours with solid and cystic components.

\section{METHODOLOGY}

This study was done in the Dapartment of Radiology, Obstetrics and Gynecology Hospital of Fudan University, China, from January to December 2017. Forty-four patients with malignant ovarian tumours confirmed by clinicopathology were selected as a malignant group. At the same time, 44 cases of benign ovarian tumours confirmed by clinic pathology were selected as a benign 
group. Inclusion criteria of malignant group and benign group in this study were ovarian masses found by pelvic and abdominal imaging examination. Exclusion criteria of two groups were endometriosis and pelvic inflammatory diseases found by relevant examination; those with chronic diseases such as complicated primary hypertension, diabetes, etc., as well as those with abnormal liver, kidney and heart functions, tumors in other organs found by relevant examination, and those with family history of cancer. The study was approved by the hospital ethics committee, and all patients volunteered to participate in the study.

All patients were scanned before surgery. The patients were told to keep a stable breath before examination, and then conventional MRI plain scans, enhanced scans and DWI scans were performed. MR scanner adopts Signa 1.5T superconductive MRI, and coil uses 8-channel phased array coils. The conventional cross section T1WI scan parameters with TR:TE at $220 / 8 \mathrm{~ms}$, scan matrix $320 \times 224$, field of view $80 \mathrm{~cm}$. T2WI fat suppression scan parameters were TR:TE 6666/88 ms, scan matrix $320 \times 224$, field of view $80 \mathrm{~cm}$. Coronal plane T2WI fat suppression scan parameters were TR:TE 6666/1100 $\mathrm{ms}$, scan matrix $320 \times 224$, field of view $1000 \mathrm{~cm}$. Gadopentetate dimeglumine was administered through the cubital vein based on the dose of $0.1 \mathrm{mmol} / \mathrm{kg}$, and then enhanced scans were performed from the above three views. DWI scan parameters were TR:TE 3000/55 ms, matrix $128 \times 128$, field of view $100 \mathrm{~cm}$, cross-sectional slice thickness $8 \mathrm{~mm}$, slice gap $1 \mathrm{~mm}$; the slice thickness was adjusted to $6 \mathrm{~mm}$ during the scanning from the sagittal view. All images were analysed by two experienced radiologists. The relevant data were transferred to the workstation and the threshold was adjusted so that both the normal and the diseased ovaries were within the threshold. The apparent diffusion coefficient and the exponential apparent diffusion coefficient of solid and cystic lesions were reconstructed. In the malignant group, the centres of solid and cystic lesions were taken as regions of interests (ROI) with a size of $100-200 \mathrm{~mm}^{2}$ and these regions were measured three times. Apparent diffusion coefficient (ADC) and exponential apparent diffusion imaging (eADC) of solid lesions of two groups were recorded.

The minimum sample size of statistical test was estimated by Ssize software. All experimental data in this study were statistically analysed with SPSS version 22. Quantitative variables, such as ADC, eADC etc. were expressed as mean \pm standard deviation, and independent samples t-test was used. Qualitative variables were expressed as frequencies with percentages. Results were considered significant at $p<0.05$.

\section{RESULTS}

In the malignant-tumors group, the age ranged from 37 to 66 years with a mean age of $53.61 \pm 1.75$ years; the number of lesions was 1 to 3 lesions, mean $1.38 \pm 0.26$ lesions. The pathological types were $20(45.45 \%)$ cases of serous cystadenocarcinoma, $7(15.91 \%)$ cases of mucinous cystadenocarcinoma, $5(11.36 \%)$ cases of ovarian epithelial carcinosarcoma, $4(9.09 \%)$ cases of ovarian endometrioid adenocarcinoma, and 8 (18.18\%) cases with metastatic carcinoma.

In benign-tumor group, the age ranged from 36 to 67 years with a mean age of $54.05 \pm 1.96$ years. Pathological types were $6(13.64 \%)$ cases with thecomas, 8 $(18.18 \%)$ cases with endometriosis, $16(36.36 \%)$ cases with corpus luteum cysts, $7(15.91 \%)$ cases with serous cystadenoma, $4(9.09 \%)$ cases with mucinous cystadenoma, and $3(6.82 \%)$ cases with mature teratomas.

In malignant group, the $\mathrm{T} 1 \mathrm{WI}$ sequence showed that 35 $(79.55 \%)$ cases of solid lesions were equal signals (Figure 1-a); $4(9.09 \%)$ cases were equal and slightly higher mixed signals (Figure 2-a); $3(6.82 \%)$ cases were slightly higher signals, and $2(4.55 \%)$ cases were high signals. T2WI fat suppression sequence showed that 33 $(75.00 \%)$ cases of solid lesions were slightly higher signals (Figure 1-b) and $11(25.00 \%)$ cases were equal and high mixed signals (Figure 2-b). The DWI showed that $41(93.18 \%)$ cases of solid lesions were high signals (Figure 1-c) and $3(6.82 \%)$ cases were high and low mixed signals (Figure 2-c). The T1WI showed that 39 $(88.64 \%)$ cases of cystic lesions were low signals (Figures 1-a and 2-a) and $5(11.36 \%)$ cases were slightly higher signals and low mixed signals. The T2Wl fat suppression sequence showed that $36(81.82 \%)$ cases of cystic lesions were significant high signals (Figures 1$\mathrm{b}$ and $2-b)$ and 8 (18.18\%) cases were high signals and slightly higher mixed signals. DWI showed that 28 $(63.64 \%)$ cases of cystic lesions were slightly higher signals (Figure 2-c) and $16(36.36 \%)$ cases were low signals (Figure 1-c).

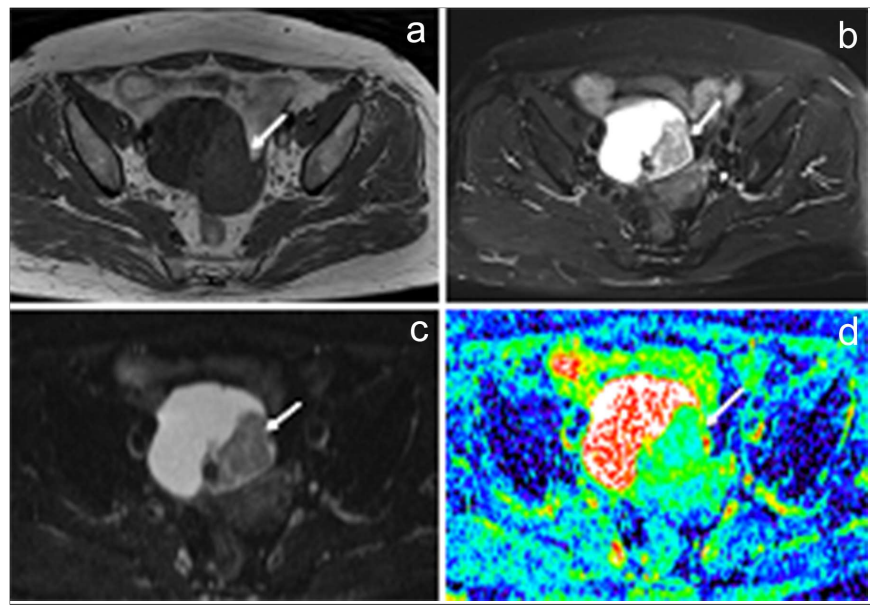

Figure 1: (a - d) Typical case 1: A female patient, 54 years old, ovarian serous cystadenocarcinoma. (a) Solid and cystic masses in the right appendage area, with equal signals (arrows) on the T1WI. (b) The T2WI fat suppression sequence showed solid and cystic lesions. (c) Solid lesions on the DWI were significant high signals. Cystic lesions on the DWI were equal signals and slightly higher mixed signals. (d) The ADC of solid lesions in the ROI was approximately $(1.15 \pm 0.14) \times 10^{-3} \mathrm{~mm}^{2} / \mathrm{s}$ 


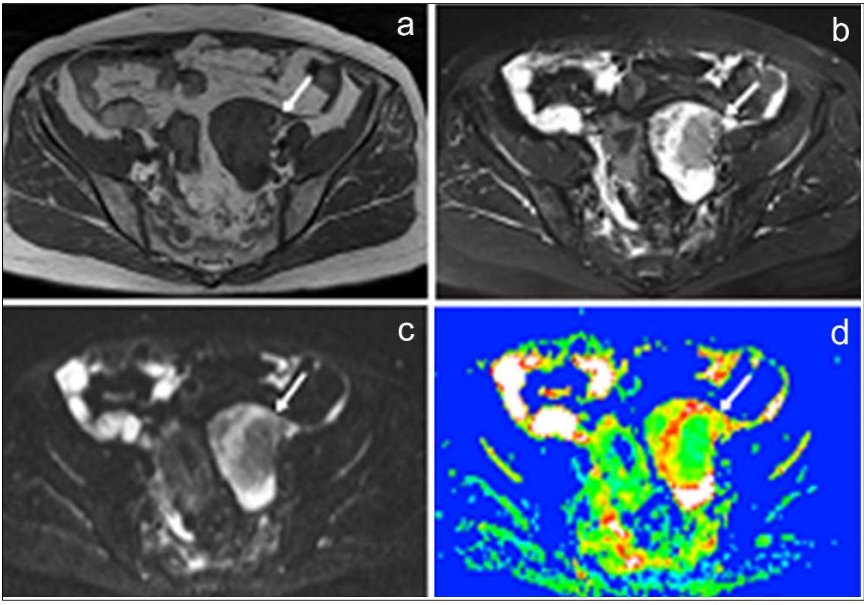

Figure 2: $(a-d)$ Typical case 2: A female patient, 52 years old, ovarian metastatic carcinoma. (a) Solid and cystic masses in the right appendage area, with low signals (arrows) on the T1WI. (b) The T2WI fat suppression sequence showed solid and cystic lesions. (c) Solid lesions on the DWI were high signals. Cystic lesions on the DWI were equal signals and high mixed signals. (d) The ADC of solid lesions in the ROI was approximately (1.39 $\pm 0.21) \times 10^{-3} \mathrm{~mm}^{2} / \mathrm{s}$.

Table I: Comparison of ADC and eADC values of solid spaceoccupying lesions in benign and malignant tumours $\left(\times 10^{-3} \mathrm{~mm}^{2} / \mathrm{s}\right)$.

\begin{tabular}{l|c|cc|cc}
\hline Groups & $\mathrm{n}$ & \multicolumn{2}{|c|}{ Solid ADC } & \multicolumn{2}{c}{ Solid eADC } \\
\cline { 3 - 6 } & & Mean \pm SD & $\mathrm{p}$-value & Mean \pm SD & $\mathrm{p}$-value \\
\hline Benign group & 44 & $1.49 \pm 0.39$ & $<0.001$ & $189.67 \pm 38.58$ & $<0.001$ \\
Malignant group & 44 & $0.95 \pm 0.13$ & & $261.48 \pm 43.40$ & \\
\hline
\end{tabular}

In benign group, the $\mathrm{T} 1 \mathrm{WI}$ sequence showed that 42 $(95.45 \%)$ cases of solid lesions were equal signals and $2(4.55 \%)$ cases were slightly higher signals. The T2WI fat suppression sequence showed that $39(88.63 \%)$ cases of solid lesions were low signals and $5(11.36 \%)$ cases were slightly higher signals. The DWI showed that $38(86.36 \%)$ cases of solid lesions were equal signals and $6(13.64 \%)$ cases were slightly higher signals and equal mixed signals. The T1WI showed that $36(81.82 \%)$ cases of cystic lesions were low signals and $8(18.18 \%)$ cases were slightly higher signals and low mixed signals. The $\mathrm{T} 2 \mathrm{Wl}$ fat suppression sequence showed that $42(95.45 \%)$ cases of cystic lesions were significant high signals and $2(4.55 \%)$ cases were high signals and slightly higher mixed signals. The DWI showed that 30 $(68.18 \%)$ cases of cystic lesions were slightly higher signals and $14(31.82 \%)$ cases were low signals.

Solid ADC in malignant group was lower than that in benign group and the difference was statistically significant $(p<0.001)$. Solid eADC in malignant group was higher than that in benign group and the difference was statistically significant $(p<0.001$, Table I).

\section{DISCUSSION}

Ovarian cancer easily undergoes necrosis, haemorrhage and other severe complications, endangering the lives of patients.11,12 It has no specific symptoms at the beginning of the disease and is easily overlooked on clinical examinations. Therefore, most diagnosed patients with ovarian cancer are in an advanced disease stage, and the prognosis and life quality of patients are seriously affected. ${ }^{13}$ Ovarian surface epithelial tumours can be divided into four types: pure cystic, cystic component, mixed, and solid.14,15 MRI is a common clinical imaging examination method that shows changes in ovarian morphology and changes in the internal structure of the mass, and has a high application value in the early diagnosis of ovarian diseases. ${ }^{16}$ Some studies have shown that ovarian size and structure were assessed noninvasively using pelvic MRI. ${ }^{17,18}$ However, MRI cannot determine the nature of malignant solid and cystic tumours, serous cystadenoma and other ovarian diseases. 19

Previous clinicopathological and radiographic findings showed that the masses meeting the following situations could be determined as malignant tumours: ovarian surface epithelial tumours with a diameter greater than $6 \mathrm{~cm}$, visible papillae in the cyst, a cyst wall gap greater than $3 \mathrm{~cm}$, solid components in the cyst, enhanced images in the solid parts under enhanced scans, and a complication of enlarged lymph nodes. ${ }^{20} \mathrm{~A}$ study showed that the differential diagnosis of benign and malignant tumours was difficult in ovarian tumours with multiple gaps and insufficient solid components, which may affect clinical treatment. 21

DWI is an imaging method that uses a special sequence of MR imaging to observe the microscopic diffusion motion of water molecules in living tissues. The principle is to add a gradient field respectively in front and at the back of a $180^{\circ}$ focused radiofrequency pulse on the basis of conventional SE sequences, and the two gradient fields are symmetric in position but have opposite polarities. When the water molecules disperse under the action of the gradient fields, the protons affected by transverse magnetisation are dispersed in phase and cannot be completely reaggregated, which in turn leads to signal attenuation, and abnormal signals are formed on the DWI. The diffuse image contains the comprehensive information on the changes of T2, protons and ADC values. The ADC values can quantitatively reflect the size of the water molecular expansion movement. Different tissues and different pathophysiological processes have different ADC values. The difference in ADC values can reflect the changes in the general and microscopic structures of the disease. ${ }^{22}$ A study found that ADC histograms of solid tumor components facilitated the distinction between borderline ovarian tumors and carcinoma. ${ }^{23}$

In this study, the authors found that solid ADC in malignant group was lower than benign group, and solid eADC in malignant group was higher than benign group, and the differences were statistically significant. The results of this study were consistent with those reported 
by Ahmad et al.24 The reason may be that the malignant cells had a faster proliferation rate and the cell gap was relatively small; the water molecular activity was limited, so the ADC levels were significantly lower than the normal levels. It showed that DWI could be used as a differential diagnostic method for malignant solid and cystic ovarian tumours.

Although this research was carefully carried out; but the authors are still aware of its limitations and shortcomings, as the sample size was small.

\section{CONCLUSION}

DWI, with its short scan time, is a fast, accurate and noninvasive means of examination and can be used as a basis for the clinical diagnosis of benign and malignant ovarian tumours with solid and cystic components. It has a high clinical application and promotion value.

\section{REFERENCES}

1. Pilone V, Tramontano S, Picarelli P, Monda A, Romano M, Renzulli M, et al. Giant mucinous ovarian borderline tumor. A good lesson from an asymptomatic case. Int J Surg Case Rep 2018; 50:25-7.

2. Jeong D, Hakam A, Abuel-Haija M, Chon HS. Ovarian microcystic stromal tumor: Radiologic-pathologic correlation. Gynecol Oncol Rep 2018; 25:11-4.

3. Nishio N, Kido A, Kataoka M, Kuwahara R, Nakao K, Kurata Y, et al. Longitudinal changes in magnetic resonance imaging of malignant and borderline tumors associated with ovarian endometriotic cyst comparing with endometriotic cysts without arising malignancy. Eur J Radiol 2018; 105:175-81.

4. Chung WJ, Chung HW, Shin MJ, Lee SH, Lee MH, Lee JS, et al. MRI to differentiate benign from malignant soft-tissue tumours of the extremities: a simplified systematic imaging approach using depth, size and heterogeneity of signal intensity. Br J Radiol 2012; 85:e831-6.

5. Drake-Pérez M, Boto J, Fitsiori A, Lovblad K, Vargas MI. Clinical applications of diffusion weighted imaging in neuroradiology. Insights Imaging 2018; 9:535-47.

6. Khizer AT, Raza S, Slehria AU. Diffusion-weighted MR imaging and ADC mapping in differentiating benign from malignant thyroid nodules. J Coll Physicians Surg Pak 2015; 25:785-8.

7. Kim BR, Song JS, Choi EJ, Hwang SB, Hwang HP. Diffusionweighted imaging of upper abdominal organs acquired with multiple B-value combinations: Value of normalization using spleen as the reference organ. Korean J Radiol 2018, 19:389-96.

8. Razik A, Das CJ, Sharma S, Seth A, Srivastava DN, Mathur S, et al. Diagnostic performance of diffusion-weighted MR imaging at $3.0 \mathrm{~T}$ in predicting muscle invasion in urinary bladder cancer: utility of evaluating the morphology of the reactive tumor stalk. Abdom Radiol (NY) 2018; 43:2431-41.

9. Mukuda N, Fujii S, Inoue C, Fukunaga T, Oishi T, Harada T, et al. Bilateral ovarian tumors on MRI: How should we differentiate the lesions? Yonago Acta Med 2018; 61:110-6.

10. Duarte AL, Dias JL, Cunha TM. Pitfalls of diffusion-weighted imaging of the female pelvis. Radiol Bras 2018; 51:37-44.
11. Nili F, Nobari N, Abdollahi A. Pseudopapillary and macrofollicular microscopic growth patterns in an advanced stage ovarian dysgerminoma: A case report. Iran J Pathol 2018; 13:94-8.

12. Takeuchi M, Matsuzaki K, Tsuneyama K, Nishimura M, Takiguchi E, Harada M. Ovarian large cell neuroendocrine carcinoma associated with serous carcinoma: Correlation of pathology with MR imaging. Magn Reson Med Sci 2017; 16:273-4.

13. Lopezacevedo M, Havrilesky LJ, Broadwater G, Kamal AH, Abernethy AP, Berchuck $A$, et al. Timing of end-of-life care discussion with performance on end-of-life quality indicators in ovarian cancer. Gynecol Oncol 2013; 130:156-61.

14. Zhou Y, Irwin ML, Ferrucci LM, Mccorkle R, Ercolano EA, Li F, et al. Health-related quality of life in ovarian cancer survivors: results from the American cancer society's study of cancer survivors-I. Gynecol Oncol 2016; 141:543-9.

15. Kurman RJ, Shih IEM. The origin and pathogenesis of epithelial ovarian cancer: a proposed unifying theory. $A m \mathrm{~J}$ Surg Pathol 2010; 34:433-43.

16. Zhao C, Wu SF, Barner R. Pathogenesis of ovarian clear cell adenofibroma, atypical proliferative (borderline) tumor, and carcinoma: clinicopathologic features of tumors with endometriosis or adenofibromatous components support two related pathways of tumor development. J Cancer 2011; 2:94-106.

17. Peters NH, Patterson AJ, Horan G, Gregory D, Sala E. Assessment of ovarian movement on consecutive pelvic MRI examinations in patients with gynaecological malignancies: what is the planning organ-at-risk volume for radiotherapy? Br J Radiol 2012; 85:1407-14.

18. Boumosleh JM, Grundy SM, Phan J, Neeland IJ, Chang A, Vega GL. Metabolic concomitants of obese and nonobese women with features of polycystic ovarian syndrome. J Endocr Soc 2017; 1:1417-27.

19. Wilde RFD, Hruban RH, Maitra A, Offerhaus GJA. Reporting precursors to invasive pancreatic cancer: pancreatic intraepithelial neoplasia, intraductal neoplasms and mucinous cystic neoplasm. Diagn Histopathol 2015; 18:17-30.

20. Pultrum BB, Van EDJ, Van HW, Van HD, Kappert P, Groen H, et al. Detection of lymph node metastases with ultrasmall superparamagnetic iron oxide (USPIO)-enhanced magnetic resonance imaging in oesophageal cancer: A feasibility study. Cancer Imaging 2009; 9:19-28.

21. Takeuchi M, Matsuzaki K, Nishitani H. Diffusion-weighted magnetic resonance imaging of ovarian tumors: differentiation of benign and malignant solid components of ovarian masses. J Comput Assist Tomogr 2010; 34:173-6.

22. Zhong WJ, Guo DJ, Zhao JN, Xie WB, Chen WJ, Wu W. Changes of axial and radial diffusivities in cerebral white matter led by normal aging. Diagn Interv Imaging 2012; 93:47-52.

23. Mimura R, Kato F, Tha KK, Kudo K, Konno Y, Oyamamanabe N, et al. Comparison between borderline ovarian tumors and carcinomas using semi-automated histogram analysis of diffusion-weighted imaging: focusing on solid components. Jpn J Radiol 2016; 34:1-9.

24. Ahmad KA, Abdrabou A. The significance of added ADC value to conventional MR imaging in differentiation between benign and malignant ovarian neoplasms. Egypt $J$ Radiol Nucl Med 2014; 45:997-1002. 